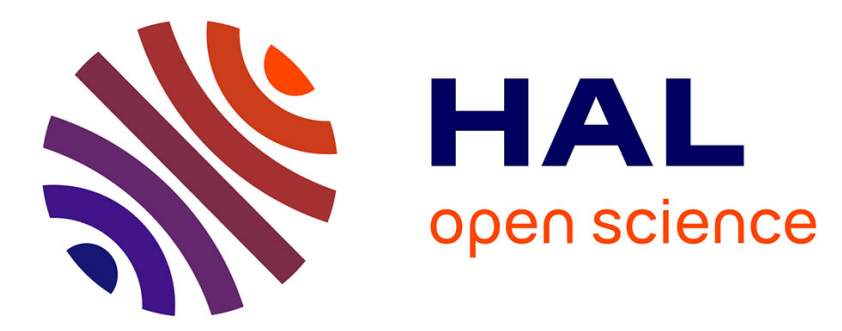

\title{
Mise en évidence d'évènements climatiques rapides par les faunes de mollusques terrestres des Loess Weichseliens de Nussloch (Allemagne)
}

Olivier Moine, Denis Didier Rousseau, Pierre Antoine, Christine Hatté

\section{- To cite this version:}

Olivier Moine, Denis Didier Rousseau, Pierre Antoine, Christine Hatté. Mise en évidence d'évènements climatiques rapides par les faunes de mollusques terrestres des Loess Weichseliens de Nussloch (Allemagne). Quaternaire, 2002, 13 (3), pp.209-217. 10.3406/quate.2002.1713 . hal-02496969

\section{HAL Id: hal-02496969 \\ https://hal.science/hal-02496969}

Submitted on 19 Oct 2020

HAL is a multi-disciplinary open access archive for the deposit and dissemination of scientific research documents, whether they are published or not. The documents may come from teaching and research institutions in France or abroad, or from public or private research centers.
L'archive ouverte pluridisciplinaire HAL, est destinée au dépôt et à la diffusion de documents scientifiques de niveau recherche, publiés ou non, émanant des établissements d'enseignement et de recherche français ou étrangers, des laboratoires publics ou privés. 
Quaternaire, 13, (3-4), 2002, p. 209-217.

\title{
MISE EN ÉVIDENCE D’ÉVÉNEMENTS CLIMATIQUES RAPIDES PAR LES FAUNES DE MOLLUSQUES TERRESTRES DES LOESS WEICHSELIENS DE NUSSLOCH (ALLEMAGNE)
}

\author{
Olivier MOINE', Denis-Didier ROUSSEAU ${ }^{1.2,}$ \\ Pierre ANTOINE ${ }^{3}$ et Christine HATTÉ ${ }^{4}$
}

\begin{abstract}
RÉSUMÉ
Une étude malacologique à haute résolution ( 1 échantillon pour $10 \mathrm{~cm}$ ) a été entreprise sur la séquence lœssique de Nußloch dans la vallée du Rhin (Allemagne). Ce travail a pour but la recherche de variations rapides du climat et/ou de l'environnement en milieu continental. Entre 19 et $31 \mathrm{ka}$ cal., la dilatation sédimentaire est exceptionnelle $(\sim 10 \mathrm{~m})$, et des variations importantes de l'indice de diversité et de l'abondance ont été mises en évidence et indiquent que des changements rapides du climat global, de l'ordre du millier d'années, ont eu lieu durant cette période. Ces variations sont mises en corrélation avec les changements lithologiques et les variations de l'indice granulométrique, caractérisant la dynamique éolienne, déterminés sur la même séquence. Les variations des proxies indiquent que la mise en place de chacun des neuf doublets gley-loess identifiés était accompagnée de trois phases climatiques successives. Ceci indique donc une grande sensibilité des environnements lœssiques aux variations climatiques millénaires.
\end{abstract}

Mots-clés : Changements climatiques rapides, Mollusques terrestres, Lœss, Weichsélien, Nußloch, Allemagne.

\begin{abstract}
EVIDENCE OF RAPID CLIMATIC EVENTS BY THE TERRESTRIAL MOLLUSC FAUNA OF THE WEICHSELIAN LOESS FROM NUSSLOCH (GERMANY)

A high resolution malacological study ( 1 sample for $10 \mathrm{~cm}$ ) realised on the loess sequence of Nußloch (Germany) in the Rhine valley has yielded its first results. The aim of this work is the search of rapid variations of climate and/or environment in continental domain. Between 19 and $31 \mathrm{ky}$ cal. BP, the sedimentological dilatation is exceptional $(\sim 10 \mathrm{~m})$, and strong variations in both the diversity index and the abundance are observed, and show that rapid global climate changes, at a millennial-time scale, occurred during this period. These variations are correlated with both the lithological changes and the grain size index, which characterises the aeolian dynamics, determined in the same sequence. The variations of the proxies indicate that the deposition of each of the nine identified gley-loess doublets is by three successive climatic changes. Thus, this indicate a great sensibility of loess environments to millennial climatic changes.
\end{abstract}

Key-words : Rapid climatic changes, Terrestrial molluscs, Loess, Weichselian, Nußloch, Germany.

\section{INTRODUCTION}

Depuis une vingtaine d'années, différents types d'événements rapides ont été mis en évidence d'après l'étude des sédiments marins de l'Atlantique Nord et les calottes de glace du Groenland durant le dernier cycle climatique (Dansgaard et al., 1982; Heinrich, 1988;
Bond et al., 1993; Bond et al., 1997). La présence de variations rapides dans les différents indices climatiques mesurés est principalement associée à des décharges d'icebergs qui affectent la formation des eaux profondes de l'Atlantique Nord (Bond et Lotti, 1995; Vidal et al., 1997), et par conséquent influencent le climat général européen (Broecker et al., 1985). En domaine continen-

\footnotetext{
1 Paléocnvironnements \& Palynologie, Institut des Sciences de l'Evolution (UMR CNRS 5554), Université Montpcllier II, casc 61, place E. Bataillon, 34095 Montpellicr Cedex 5, France, E-mail : omoine@isem.univ-montp2.fr. Denis.Rousseau@dstu.univ-montp2.fr

2 Lamont-Doherty Earth Observatory of Columbia University, Palisades, NY 10964, USA.

3 UMR CNRS 8591 - Laboratoire de Géographie Physique. 1. Place Aristide Briand. 92195 Meudon cedex, France, E-mail : Pierre.Antoine @cnrs-bellevue.fr

4 Laboratoire des Sciences du Climat et de l'Environnement, UMR 1572 CEA/CNRS. Bât 12. Domaine du CNRS. Avenue de la Terrasse, F-91198 Gif-sur-Yvette Cedex. France, E-mail : Christine.Hatte@lsce.cnrs-gif.fr
} 


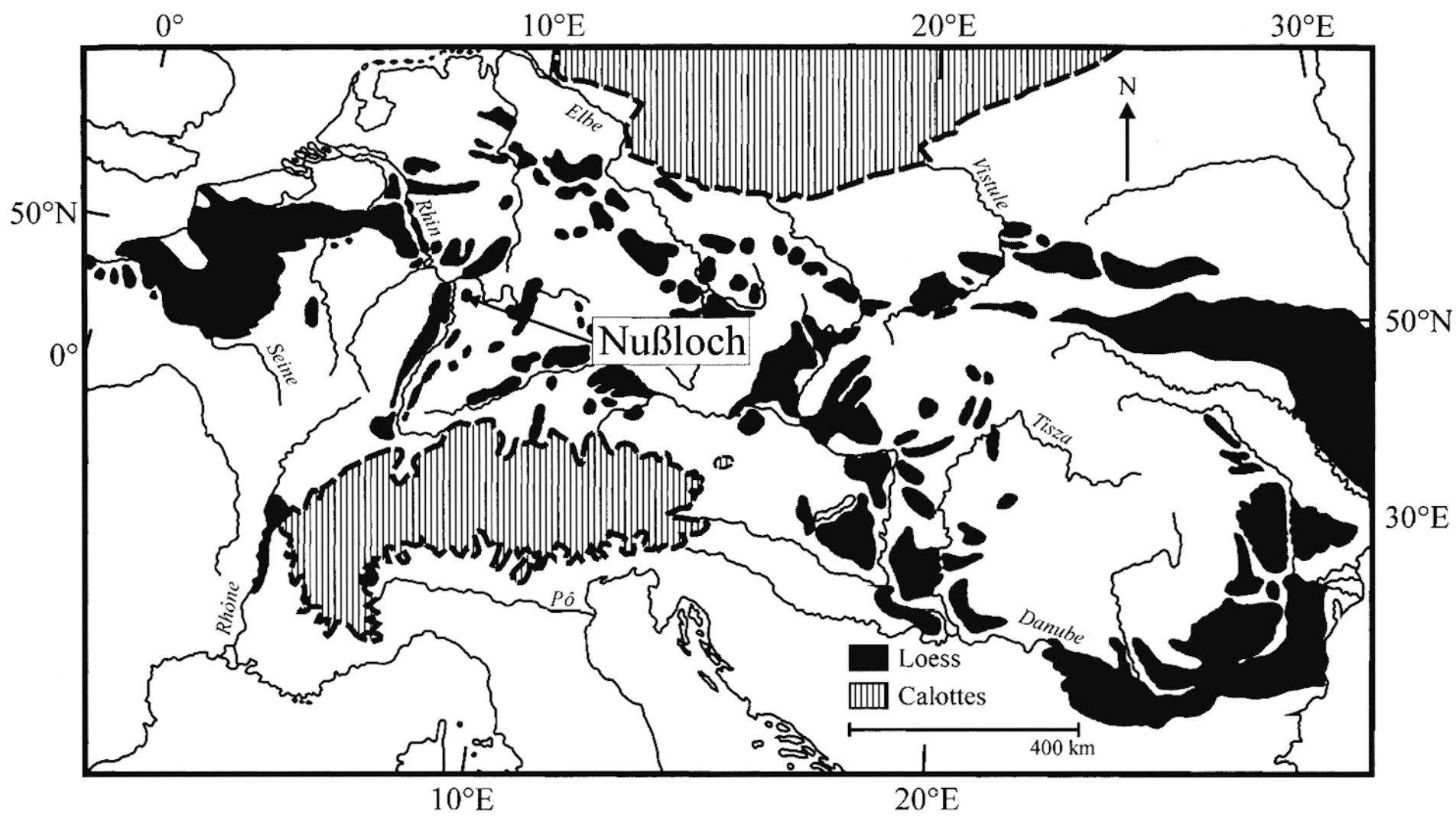

Fig. 1: Localisation du site de Nußloch et dépôts de lœss en Europe occidentale lors de l'extension maximale des calottes glaciaires scandinave et alpine durant le dernier cycle climatique (Modifié d'après Grahmann, 1932

(calottes) et Haesaerts, 1985 (lœss).

Fig. 1: Location of the Nußloch site and loess deposits in Western Europe during the maximal extent of the Scandinavian and Alpine ice-sheets during the last climatic cycle (Modified after Grahmann, 1932 (ice sheets) and Haesaerts 1985 (loess).

tal européen, les changements rapides du climat ou de l'environnement ne sont documentés, notamment pendant les stades isotopiques marins (SIM) 2 et 3, que par quelques séquences lacustres (Magny, 1995), polliniques (Guiot et al., 1993; Allen et al., 1999) et lœssiques (Vandenberghe et al., 1998).

En milieu continental, hors systèmes lacustres, les lœss constituent des dépôts de milieu ouvert se présentant sous la forme de séquences. Ainsi, dans certains sites d'Europe de l'Ouest et d'Europe de l'Est, les dépôts lœssiques ont enregistré des variations du climat pendant plus de cinq cycles climatiques (Kukla, 1977; Puisségur, 1978; Lautridou et al., 1985). Durant le Pléistocène supérieur, derniers 130000 ans, d'importants dépôts éoliens ont été mis en place en Europe dans une zone située entre les calottes fenno-scandinave au nord et alpine au sud, et s'étendant de la Bretagne à l'Ukraine (Figure 1) (Grahmann, 1932; Haesaerts, 1985). Le site de Nußloch (Allemagne), échantillonné pour cette étude, est situé à quelques kilomètres au sud de Heidelberg sur la bordure ouest du plateau de l'Odenwald bordant le flanc est de la vallée du Rhin (Figure 1). Au Dernier Maximum Glaciaire, le site était à peu près équidistant des deux calottes de glace évoquées ci-dessus. Dans la séquence $P 3,9,5 \mathrm{~m}$ de lœss se sont déposés entre 31 et 19 ka BP (Figure 2). La dilatation de cette séquence, presque inédite en Europe de l'ouest pour cette période, est donc très favorable à l'étude des variations climatiques rapides en milieu continental durant le stade isotopique marin 2 (Antoine et al., 2001). Les dépôts sont composés d'une alternance de gleys de toundra et de lœss (Sommé et al., 1986; Antoine et al., 1999; Antoine et al., 2001) particulièrement nette à Nußloch (Figure 2). La série dilatée débute par un lœss calcaire homogène (22), dont la moitié supérieure (22b) présente des traces de gélifluxion, et se termine par des lœess calcaires homogènes ( 36 et 38 ). Les lœss 24 à 34 sont constitués de fine lamines séparées par des niveaux de microfentes de cryo-dessiccation, indiquant un environnement de dépôt plus humide que celui des lœss homogènes. Les lœss signalent des périodes à forte activité éolienne contrairement aux gleys de toundra indiquant un affaiblissement voire un arrêt du dépôt de particules accompagné d'une humidité plus importante et de conditions climatiques dépréciées. Les quatre premiers gleys de toundra sont cryoturbés et trois $(23,26$ et 28$)$ sont relativement épais, alors que les quatre derniers sont moins développés et réguliers, indiquant une moindre humidité lors de leur formation (Antoine et al., 2001).

En Europe de l'ouest, les corrélations entre différentes séquences du Pléistocène supérieur sont facilitées par la présence d'un certain nombre de marqueurs. Les 
forts dépôts lœssiques de cette période surmontent un horizon de sol brun (horizon Bw de Cambisol) appelé "Löhner Boden" en Allemagne (Frechen, 1999), et daté à $34 \pm 3,2$ ka BP à Nußloch (Zöller et al., 1988) et 34,1 $\pm 4,3 \mathrm{ka}$ BP à Achenheim (Rousseau et al., 1998). Le sommet de ce niveau est affecté par des phénomènes de gélifraction dans le nord de la France et la Belgique (fentes en coins), et à Achenheim (fentes de gel) (Antoine et al., 2001). Un second marqueur est le tuf d'Eltviller situé vers le sommet de la séquence et daté par luminescence optique stimulée à ca. 19,5 $\pm 2,3 \mathrm{ka}$ (Antoine et al., 2001). Le troisième marqueur est un horizon perturbé appelé horizon à langues de Nagelbeek présent dans la majorité des sites d'Europe de l'ouest et sus-jacent au tuf d'Eltviller. Les datations issues du sol brun, de l'horizon de Nagelbeek et du tuf montrent ainsi que ces $9,5 \mathrm{~m}$ de dépôts éoliens se sont mis en place en un peu plus de 10000 ans.

Enfin, hormis les différents paramètres sédimentologiques, géochimiques et géophysiques qui peuvent y être mesurés, les lœss renferment de grandes quantités de mollusques terrestres. Sensibles au climat et à l'environnement, ils sont considérés comme d'excellents indicateurs paléobioclimatiques (Puisségur, 1976; Rousseau, 1987; Rousseau et al., 1990). La dynamique des vents d'ouest à nord-ouest étant plus intense durant les derniers stades glaciaires (COHMAP Members, 1988), les variations climatiques rapides, enregistrées dans l'Atlantique Nord, sont susceptibles d'avoir influencé le climat et l'environnement du continent européen adjacent et conduit à des variations synchrones des malacofaunes de Nußloch.

\section{MATÉRIEL ET METHODE}

Les prélèvements malacologiques, les mesures du $\delta^{13} \mathrm{C}$ de la matière organique des lœss et de l'indice granulométrique (IGR $=20$ à $50 \mathrm{~mm} /<50 \mathrm{~mm}$ ) ont été effectuées dans le cadre des projets européens BIMACEL (Biological and Magnetic Records and Dating of Climate Changes in Western European Loss series EV5V-0298) et EOLE (Enregistrement des Evénements Eoliens dans les séquences Lœssiques du dernier cycle climatique en Europe, Programme ECLIPSE). La série synthétique, définie à partir des trois profils P1, P2 et P3, couvre de façon discontinue un intervalle compris entre environ 130 et $19 \mathrm{ka} \mathrm{BP}$ (Antoine et al., 2001). L'étude menée ici s'est restreinte à l'intervalle 31 - 19 ka BP.

Les valeurs de l'indice granulométrique et du $\delta^{13} \mathrm{C}$ de la matière organique des lœss ont été mesurées sur le profil P2. Les prélèvements malacologiques proviennent du profil P3, distant de quelques dizaines de mètres, et dont la stratigraphie et quasiment identique à celle de P2 . Le site étant localisé dans une carrière en activité, ces deux profils ont aujourd'hui disparu. Le sédiment a été prélevé en continu tous les $10 \mathrm{~cm}$ par échantillons de 10 litres chacun. Différentes unités stratigraphiques ont été distinguées dans $\mathrm{P} 2$ et numérotées de 21 à 38 (Antoine et al., 2001). Cette numérotation a été appliquée à $P 3$ en incluant quelques subdivisions, soit à cause d'un chan- gement latéral de faciès par rapport à $\mathrm{P} 2$ (unité 31 ), soit en raison d'un changement lithologique interne marquant (unités 22 et 24). Les 95 échantillons ont été lavés, tamisés à $0,42 \mathrm{~mm}$, et les coquilles identifiées et comptées (Figure 2). L'abondance des assemblages est donnée par les comptages, et les indices de diversité $(\mathrm{H})$ et d'équitabilité (R) de Shannon-Weaver (Blondel, 1995) ont été calculés à l'aide de leurs formules respectives :

$$
\mathbf{H}=-\overline{\bar{\Sigma}}\left(p_{k} \log _{2} p_{k}\right) \text { et } \mathbf{R}=-\underline{\Sigma}\left(p_{k} \log _{2} p_{k}\right) / \log _{2} \mathbf{n}
$$

Où pk représente la fréquence de l'espèce $\mathrm{k}$, et $\mathrm{n}$ le nombre d'espèce dans l'assemblage. Plus la valeur de l'équitabilité relative à un assemblage est élevée, plus la distribution des effectifs entre les différentes espèces est équitable. L'assemblage est alors considéré en équilibre avec son milieu. Par la suite, on parlera de diversité lorsque l'on se référera à l'indice de diversité.

Des comparaisons avec la lithologie, le $\delta^{13} \mathrm{C}$ de la matière organique des lœss et l'indice granulométrique ont aussi été entreprises pour étudier l'évolution simultanée des différents indicateurs paléoclimatiques. Ces comparaisons ont nécessité la normalisation des profondeurs de P2 en profondeurs de P3, à l'aide du logiciel AnalySeries (Paillard et al., 1996), en utilisant les limites lithologiques comme points de référence.

\section{RÉSULTATS}

Au sein de la séquence éolienne dilatée de Nußloch, la composition spécifique des assemblages montre une relative monotonie avec près de la moitié des espèces identifiées (Trichia hispida, Succinea oblonga elongata, Pupilla muscorum, Clausilia parvula, Arianta arbustorum et Vallonia costata), présentes dans plus de $80 \%$ des assemblages (Figure 2). Ces associations malacologiques caractérisent une faune à Pupilla (Lozek, 1968) qui représente le pôle médian des environnements de steppes lœessiques, ni très froid, ni très sec. De plus, la présence dans des proportions équivalentes de Trichia hispida, Succinea oblonga elongata, et Pupilla muscorum, est caractéristique d'un faciès de loess humide (Lozek, 1968). Seule l'unité 24a, dominée par Succinea oblonga elongata, Columella columella et Vertigo genesii, présente une association qui se rapproche d'une faune à Columella caractérisant un environnement de toundra plus froid et plus humide que le précédent (Puisségur, 1977).

Les variations d'abondance des espèces sont pour la plupart synchrones et abruptes, intervenant souvent entre deux échantillons adjacents (Figure 2). La séquence a ainsi été subdivisée en six zones $(Z)$ sur la base des trois indices écologiques : abondances spécifique et totale, diversité et équitabilité. Les différentes zones ont été individualisées à l'aide de combinaisons de ces trois paramètres (Figure 3). $\mathrm{Zl}$ et $\mathrm{ZA}$ ont une abondance élevée, une diversité et une équitabilité moyennes. $Z 3$ et $\mathrm{Z5}$ ont une abondance faible, une diversité et une équitabilité élevées (sauf au sommet de Z5). Z2 a une abondance élevée, une diversité moyenne et une équitabilité faible. 


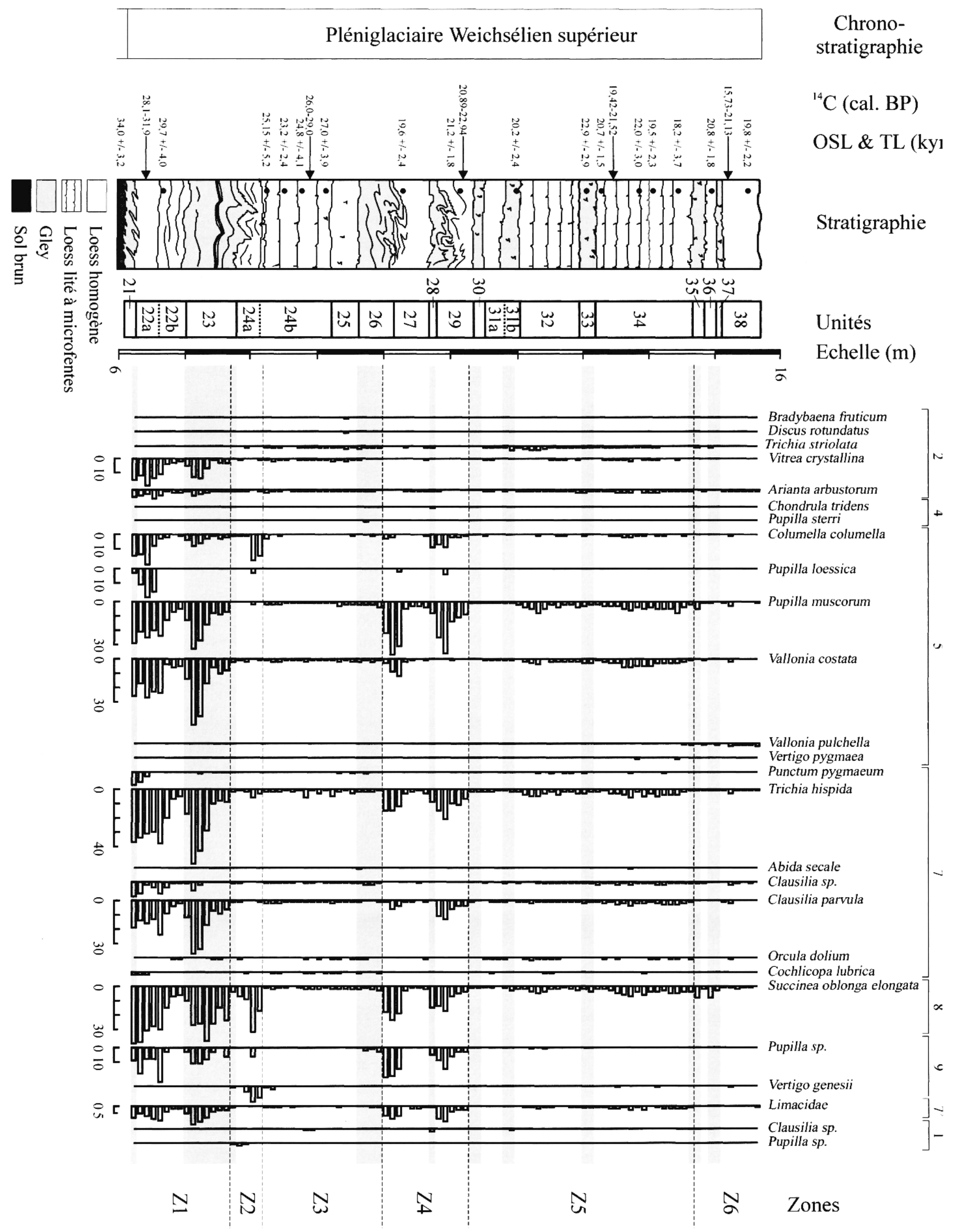


Z6 a une abondance, une diversité et une équitabilité faibles. Dans Z1, Z2 et Z4, les abondances sont élevées et affectées de variations abruptes, importantes et relativement régulières. Toutefois, des variations de moindre amplitude sont aussi observables dans Z3, Z5 et Z6. Les espèces Vitrea crystallina, Arianta arbustorum, Clausilia dubia et Punctum pygmaeum sont beaucoup mieux représentées dans $Z 1$ que dans $Z 2$ et $Z 4$, indiquant que la végétation y était plus développée, en particulier à sa base. De même, Z2, et en particulier l'unité 24a, est dominée par Columella columella, Succinea oblonga elongata et Vertigo genesii, ce qui l'individualise clairement de $\mathrm{Zl}$.

Les enregistrements de l'abondance, de la diversité et de l'équitabilité ont été placés en parallèle avec la lithologie, le $\delta^{13} \mathrm{C}$ de la matière organique des lœss et l'indice granulométrique IGR (Figure 3). Les valeurs de l'abondance sont globalement inférieures à 250 individus par échantillon, sauf pour cinq groupes d'assemblages, numérotés de 1 à 5 , où elles peuvent atteindre plusieurs milliers d'individus, dans $\mathrm{Z1}, \mathrm{Z} 2$ et $\mathrm{Z4}$.

\section{DISCUSSION}

Les valeurs de l'équitabilité à Nußloch ont été comparées avec celles d'échantillons de malacofaunes modernes s'échelonnant entre le nord de la Norvège et le sud de la France. Ces prélèvements ont été réalisés loin de tout bouleversement récent de la végétation d'origine anthropique, dans des milieux forestiers en équilibre avec le climat actuel (Rousseau, 1992). Les principes d'échantillonnage actuels et fossiles étant relativement similaires de telles comparaisons peuvent être faites (Puisségur, 1976). Celles-ci peuvent toutefois être biaisées par des mélanges de faunes dus à des arrêts de la sédimentation, des prélèvements d'épaisseur trop importante ou des transports post-mortem. Or, d'après la composition spécifique des assemblages, semblable à celle d'autres sites caractérisant des environnements lœssiques (Lozek, 1968; Puisségur, 1978), aucun mélange faunistique n'interviendrait dans les assemblages de Nußloch. L'équitabilité des échantillons modernes s'échelonne entre 0,73 et 0,8 , ce qui confirme qu'avec des valeurs le plus souvent supérieures ou égales à 0,7 , les malacofaunes de Nußloch sont en équilibre avec leur environnement.
Toutefois, cet équilibre ne se caractérise pas toujours de la même manière lorsque l'on tient compte aussi de la diversité (Dyduch-Falniowska, 1988). Une équitabilité et une diversité élevées (respectivement $>0,85$ et $>2,9$ ) comme dans $\mathrm{Z3}$ et $\mathrm{Z5}$, caractérisent les malacofaunes profitant au mieux des ressources de leur environnement, sans compétition entre les espèces qui ont ici des effectifs faibles. Ces assemblages prennent place durant les phases de dépôts lœssiques les plus importantes. Une équitabilité et une diversité moyennes (respectivement $0,6-0,85$ et $2-2,9$ ), comme dans $Z 1$ et $Z 4$, indiquent que les ressources sont limitées pour la malacofaune locale. Ceci interdit un développement équivalent de toutes les espèces (équitabilité réduite), et la présence de moins d'espèces (diversité réduite) probablement en raison d'une forte compétition interspécifique vis-à-vis des ressources disponibles (Dyduch-Falniowska, 1988).

Ces assemblages apparaissent durant les périodes dominées par le développement de gley. L'impact de l'amélioration climatique propice au développement des malacofaunes serait restreinte par un autre facteur du milieu, comme le manque de nourriture. Enfin, une équitabilité variable mais souvent faible $(0,17-0,87$ ou $<0.6)$ et une diversité faible $(<2)$ caractérisent des environnements très peu favorables aux malacofaunes restreignant la diversification des espèces. Le petit nombre d'espèces capables de s'adapter à ces conditions environnementales difficiles peuvent soit développer des populations importantes, comme dans $\mathrm{Z} 2$ où l'abondance est élevée, soit perdurer en communautés restreintes comme dans Z6 où l'abondance est très faible (Figure 3). Dans ces deux zones interviennent respectivement les maxima de froid (Z2), d'après les espèces présentes, et d'humidité (Z6) d'après le $\delta^{13} \mathrm{C}$ de la matière organique des lœss. La structuration des malacofaunes est donc variable en fonction des différents ensembles sédimentologiques.

Les perturbations enregistrées dans les malacofaunes et reflétées par la diversité ont déjà été mises en relation avec les variations générales du climat (Rousseau, 1992; Rousseau et al., 1993). Dans le détail, de nombreuses oscillations plus rapides que celles décrites précédemment sont visibles sur la courbe de diversité. De plus, à chaque gley de toundra (Figure 3, unités 24a, 27, 30, $31 \mathrm{~b}, 33,35$ et 37) est associée une baisse de diversite, indiquant une dégradation climatique, qui se termine par

Fig. 2: Diagramme d'abondance des espèces identifiées dans la séquence P3 de Nußloch. Afin de faciliter la lecture, les valeurs de l'abondance sont représentées par la racine carrée des comptages absolus. Les espèces ont été rassemblées par groupes écologiques (Puisségur, 1976) : 2=Semi-forestiers ; 4=Steppiques ; 5=Terrain découvert ; 7=Mésophiles ; 8=Hygrophiles ; 9=Palustres ; 7'=Limacidae ; I=Indéterminés. Log stratigraphique de la séquence P3, et numérotation des unités lithologiques selon Antoine et al. 2001 et de leurs subdivisions (cette étude). La distinction des malacozones, numérotées 21 à 26, est basée sur les variations d'abondance. Les zones grisées signalent les gleys de toundra.

Fig. 2: Abundance diagram of the identified species in the P3 sequence of Nußloch. In order to ease the reading of the diagram, the abundance values are expressed by the square root of the absolute counts. The species are gathered in ecological groups as defined by Puisségur (1976): 2=Open woodland; 4=Steppe; 5=Grassland; 7=Mesic; 8=Damp; 9=Wetland; 7'=Slugs; $1=$ Undetermined.

Stratigraphy of the P3 sequence and labelling of the lithological units from Antoine et al. 2001, and of their subdivisions (this study). Characterization of mollusc zones, labelled from 21 to 26 , follows the abundance variations. The grey zones indicate the tundra gleys. 


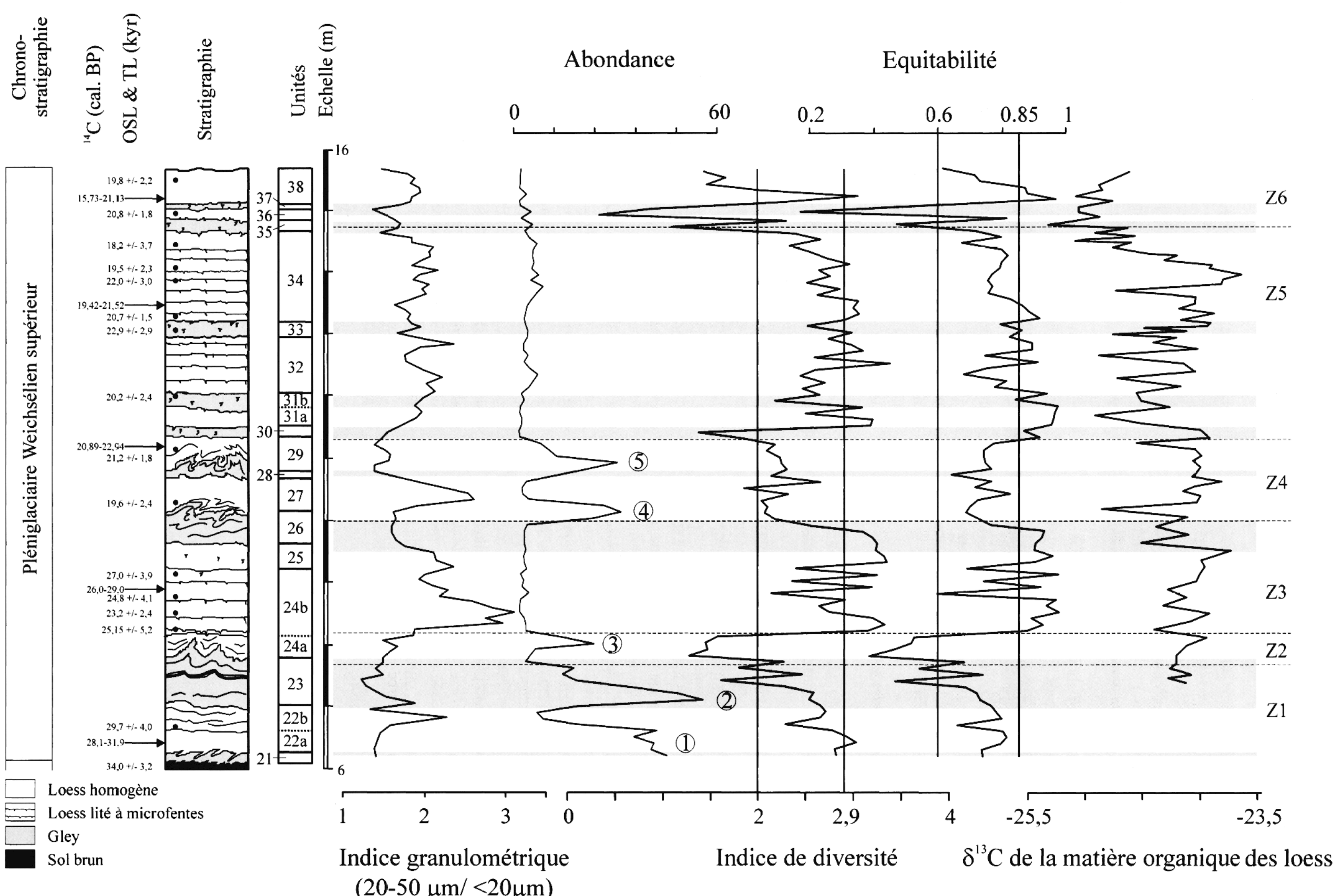


une chute brutale dans la partie sommitale des gleys. Or, les alternances gleys de toundra - lœss sont rencontrées dans tous les dépôts du Pléistocène supérieur d'Europe du nord-ouest (Vandenberghe et al., 1998; Frechen, 1999; Antoine et al., 2001). La mise en place de ces gleys relèverait d'oscillations du climat général de courte période ( $<1 \mathrm{ka}$ ) (Antoine et al., 2001; Rousseau et al., 2002). Ces horizons reflètent des périodes de forte baisse, voire d'arrêt, de la sédimentation éolienne. Ils indiquent des conditions beaucoup plus humides, justifiées par des phénomènes de décalcification et de redistribution des carbonates, de réduction et redistribution du fer, et d'un léger enrichissement en matière organique (Van Vliet-Lanoë, 1987). La présence de gley souligne également des basses températures justifiée en outre par des traces de cryoturbation et de coins de glaces caractérisant l'installation d'un permafrost (Van Vliet-Lanoë, 1987; Vandenberghe et al., 1998). Ces observations sur les malacofaunes sont donc en adéquation avec les interprétations basées sur les paramètres sédimentologiques. Les variations de l'indice de diversité des malacofaunes le long du profil reflètent donc l'impact de la cyclicité climatique globale à Nußloch.

Cependant, la courbe d'abondance montre des variations qui sont bien différentes de celles des autres indices écologiques utilisés. Tout d'abord, les cinq maxima d'abondance sont synchrones de valeurs faibles de l'indice granulométrique, qui indiquent une dynamique éolienne plus faible (Xiao et al., 1995). Or, étant donné les effectifs moyens relevés dans les assemblages hors maxima (environ 250 individus), l'obtention de telles abondances (plus de 2000 individus) par le biais d'une accumulation de coquilles due seulement à un arrêt total de la sédimentation impliquerait une durée trop importante compte tenu du cadre chronologique dont nous disposons (Figure 3). Un mécanisme biologique et/ou environnemental ayant favorisé l'expansion des populations de mollusques, est donc à envisager pour expliquer l'origine de ces maxima d'abondance.

D'un point de vue sédimentologique, le maxima 2 est situé dans un gley (unité 23), alors que les quatre autres interviennent dans des niveaux perturbés directement sus-jacents à des gleys de toundra (21-22a, 24a, 26 et 28). Aucun niveau cryoturbé non accompagné d'un maximum d'abondance n'est visible dans le profil. Leurs occurrences semblent donc liées. Selon Antoine (2001), les unités 21 et 23 à 33 se sont développées dans un contexte globalement plus humide. Cette humidité, accompagnant aussi la formation des gleys de toundra, associée au froid a permis l'installation d'un permafrost.
L'hypothèse de réchauffements périodiques intenses intervenant au sommet des gleys de toundra expliquerait alors les explosions démographiques des malacofaunes et la fonte du permafrost conduisant à la perturbation des sols. En effet, d'après les données micromorphologiques (loess homogène sans trace de météorisation) (Antoine et al., 2001), et les reconstructions de précipitations d'après les mesures du $\delta^{13} \mathrm{C}$ de la matière organique des loess (Hatté et al., 1998), une aridification importante intervient au sommet de la séquence dans les unités 35 à 37. Ce résultat n'est pas incompatible avec l'humidité locale élevée indiquée par $\delta^{13} \mathrm{C}$ lui-même (cf. climat actuel d'Europe septentrionale). L'absence de maxima d'abondance et de niveau perturbé peut être notée alors que les valeurs de l'indice granulométrique sont aussi faibles qu'à la base de la coupe. Une aridité trop importante du milieu empêcherait donc le développement démographique des malacofaunes même en cas d'amélioration thermique. Toutefois, cet élément n'explique pas l'absence de perturbation et de maximum d'abondance au sommet des unités $30,31 \mathrm{~b}$ et 33 . On peut toutefois supposer que des améliorations climatiques très courtes ou peu importantes ont eu lieu, mais trop faibles pour provoquer l'explosion démographique et la fonte complète du permafrost. En ce qui concerne le maximum 2, d'après la stratigraphie du profil P4 (Antoine, non publié), l'unité 23 , dans laquelle il intervient, est formée par deux gleys séparés par un lœss. Le gley de base est perturbé, impliquant que le maximum 2 est probablement lié à une amélioration climatique rapide intervenue après la formation du premier gley de cette unité, mais qui n'aurait pas été enregistrée dans la séquence P3. Ceci suggère donc que finalement il n'y aurait qu'un même mécanisme pour expliquer tous les maxima d'abondance, que des analyses plus détaillées devraient permettre d'expliquer.

\section{CONCLUSION}

Les premiers résultats de l'analyse malacologique de la séquence de Nußloch (Allemagne) indiquent que les malacofaunes réagissent de manières diverses aux différents types de changements climatiques ayant eu lieu localement. Le découpage de la séquence en zones à l'aide des paramètres écologiques indique que les périodes à forts dépôts lœssiques sont plus propices au bon développement des malacofaunes que les gleys de toundra. Plus en détails, l'alternance loss - gleys de toundra est soulignée par des variations de l'indice de diversité. Les variations synchrones de la lithologie et de la malaco-

Fig. 3 : Comparaison des paramètres écologiques issus de la malacofaune obtenus sur la séquence P3 et de l'indice granulométrique, calculé sur la séquence P2 pour l'intervalle 31-19 ka BP à Nußloch, (Antoine et al., ce volume) après transformation des profondeurs de $\mathrm{P2}$ en P3. Cinq maxima d'abondance ont été numérotés de 1 à 5 . Les flèches indiquent les principales tendances de la courbe de la richesse spécifique. Cf. figure 2 pour les légendes complémentaires.

Fig. 3: Comparison between the ecological indices calculated from the malacofauna on the P3 sequence and the grain size index measured on the P2 sequence for the $31-19 \mathrm{ka}$ interval at Nußloch, (Antoine et al., this volume) after the transformation of the P2 depths into $P 3$ depths using the limits of each identified unit as a reference. Five maximums of abundance are identified and labelled from 1 to 5 . The arrows indicate the main tendencies of the diversity curve. See figure 2 for further legends. 
faune ont été résumées dans le modèle hypothétique suivant. La première phase est celle de dépôt des loess en climat sec et froid, avec une dynamique éolienne renforcée, et des malacofaunes typiques des steppes lœssiques légèrement humides. La deuxième phase est celle de développement des gleys de toundra dans un contexte plus froid et plus humide (développement d'un permafrost), avec des malacofaunes similaires à celles des lœss, mais moins développées. La troisième phase est une amélioration climatique rapide caractérisée par la fonte du permafrost, lorsqu'il est présent, mais par aucun dépôt particulier associé, et par des explosions d'abondance de la malacofaune lorsque l'humidité est suffisante, et des augmentations de l'indice de diversité. Le pic d'abondance est le plus souvent suivi d'une augmentation de l'indice de diversité marquant le retour à l'environnement de steppe lossique dominant. Au sein de la séquence $\mathrm{P} 3$, mise en place sur environ $12 \mathrm{ka}$, neuf successions gley-lœss réparties de façon relativement homogène, et caractérisées par leurs malacofaunes respectives, ont été individualisées. Cette observation amène à souligner la forte sensibilité des environnements lœessiques aux variations climatiques millénaires.

\section{REMERCIEMENTS}

Nous tenons à remercier la société Heidelberg Zement AG pour nous avoir autorisé l'accès aux carrières de Nußloch, ainsi que Nicole Limondin-Lozouet et Jean Sommé pour leurs commentaires qui ont permis l'amélioration de cet article. Ce travail est une contribution ISEM 2002-026.

\section{BIBLIOGRAPHIE}

ALLEN J.R.M., BRANDT U., BRAUER A., HUBBERTEN H.-W., HUNTLEY B., KELLER J., KRAML M., MACKENSEN A., MINGRAM J., NEGENDANK J.F.W., NOWACZYK N.R., OBERHÄNSLI H., WULF S. \& ZOLITSCHKA B., 1999 - Rapid environmental changes in southern Europe during the last glacial period. Nature, 400, 740-743.

ANTOINE P., ROUSSEAU D.-D., LAUTRIDOU J.-P. \& HATTE C., 1999 - Last interglacial-glacial climatic cycle in loess-paleosol successions of north-western France. Boreas, 28, 551-563.

ANTOINE P., ROUSSEAU D.-D., ZÖLLER L., LANG A., MUNAUT A.-V., HATTÉ C. \& FONTUGNE M., 2001 - Highresolution record of the last Interglacial-glacial cycle in the Nussloch loess-paleosol sequences, Upper Rhine Area, Germany. Quaternary International, 76/77, 211-229.

ANTOINE P., ROUSSEAU D.-D., HATTÉ C., ZÖLLER L., LANG A. FONTUGNE M., \& MOINE O., ce volume - Événements éoliens rapides dans les loess du Pléniglaciaire supérieur weichselien : l'exemple de la séquence de Nussloch (Vallée du Rhin Allemangne).

BLONDEL J., 1995 - Biogéographie: Approche écologique et évolutive. Masson, Paris, 297 p.

BOND G., BROECKER W., JOHNSEN S., MC- MANUS J.F., LABEYRIE L., JOUZEL J. \& BONANI G., 1993 - Correlation between climate records from North Atlantic sediments and Greenland ice. Nature, 365, 143-147.

BOND G.C. \& LOTTI R., 1995 - Iceberg discharges into the North Atlantic on millenial time scales during the last deglaciation. Science, 267, 1005-1010.
BOND G.C., SHOWERS W., CHESEBY M., LOTTI R., ALMASI P., DEMENOCAL P., PRIORE P., CULLEN H., HAJDAS I. \& BONANI G., 1997 - A pervasive millenial-scale cycle in North Allantic Holocene and glacial climates. Science, 278, 1257-1266.

BROECKER W., PETEET D. \& RIND D., 1985 - Does the oceanatmosphere system have more than one stable mode of operation ? Nature, 315, 21-26.

COHMAP MEMBERS, 1988 - Climatic changes of the last 18,000 years : observations and model simulations. Science, 241, 1043-1052.

DANSGAARD W., CLAUSEN H.B., GUNDESTRUP N.S, HAMMER C.U., JOHNSEN S.J., KRISTINDOTTIR P.M. \& REEH N., 1982 - A new Greenland deep ice core. Science, 218, 1273-1277.

DYDUCH-FALNIOWSKA A., 1988 - Similarity, diversity and equitability of snail communities in lower mountain zone in the Tatra mountains. Folia Malacologia, 1112, 7-28.

FRECHEN M., 1999 - Upper Pleistocene loess stratigraphy in Southern Germany. Quaternary Geochronology, 18, 243-269.

GRAHMANN R., 1932 - Der Löß in Europa. Mitteilungen der Gesellschaft für Erdkunde zu Leipzig, 51, 5-24.

GUIOT J., BEAULIEU (DE) J.-L., CHEDDADI R., DAVID F., PONEL P. \& REILLE M., 1993 - The climate in Western Europe during the last Glacial/Interglacial cycle derived from pollen and insect remains. Palaeogeography, Palaeoclimatology. Palaenecology, 103, 73-93.

HAESAERTS P., 1985 - Les loess du Pléistocène supérieur en Belgique ; comparaisons avec les séquences d'Europe centrale. Bulletin de l'Association Française pour l'Etude du Quaternaire, $2-3,105-115$

HATTÉ C., FONTUGNE M., ROUSSEAU D.-D., ANTOINE P., ZÖLLER L., TISNERAT-LABORDE N. \& BENTALEB I., $1998-\delta^{13} \mathrm{C}$ variations of loess organic matter as a record of the vegetation response to climatic changes during the Weichselian Geology, 26, (7), 583-586.

HATTÉ C. ANTOINE P., FONTUGNE M. LANG A., ROUSSEAU D.-D. \& ZÖLLER L., $2001-\delta^{13} \mathrm{C}$ of loess organic matter as a potential proxy for paleoprecipitation. Quaternary Research, 55, 33-38.

HEINRICH H., 1988 - Origin and consequences of cyclic ice rafting in the Northeast Atlantic ocean during the past 130,000 years. Quaternary Research, 29, 142-152.

KUKLA G., 1977 - Pleistocene land-sea correlations. I. Europe Earth Science Reviews, 13, 307-374.

LAUTRIDOU J.-P., SOMMÉ J., HEIM J., PUISSE-GUR J.-J. \& ROUSSEAU D.-D., 1985 - La stratigraphie des loess et formations fluviatiles d'Achenheim (Alsace) : Nouvelles données bioclimatiques et corrélations avec les séquences Pléistocène de la France du Nord-Ouest. Bulletin de l'Association Française pour l'Etude du Quaternaire, 22-23, 125-132.

LOZEK V., 1968 - The loess environment in Central Europe. In : C Schultz, J.C. Frye (ed.), Loess and Related Eolian Deposits of the World, Proceedings 7th INQUA Congress, Boulder, 1965. University of Nebraska Press, Lincoln, 67-80.

MAGNY M., 1995 - Successive oceanic and solar forcing indicated by Younger Dryas and Early Holocene climatic oscillations in the Jura. Quaternary Research, 43, 279-285.

PAILLARD D., LABEYRIE L. \& YIOU P., 1996 - Macintosh program performs time-series analysis. EOS, Transactions, American Geophysical Union, 77, 379.

PUISSÉGUR J.-J., 1976 - Mollusques continentaux quaternaires de Bourgogne. Significations stratigraphiques et climatiques. Rapports avec d'autres faunes boréales de France. Mémoires géologiques de l'Université de Dijon, 3, 1-241.

PUISSÉGUR J.-J., 1977 - Les mollusques quaternaires. In: H. Laville et J. Renault-Miskovsky, Approche écologique de l'homme fossile. Bulletin de l'Association Française pour l'Etude du Quaternaire, 47 suppl., 83-85.

PUISSÉGUR J.-J., 1978 - Les mollusques des séries loessiques à Achenheim. Recherches géographiques Strasbourg, 7, 71-96. 
ROUSSEAU D.-D., 1987 - Paleoclimatology of the Achenheim series (middle and upper Pleistocene, Alsace, France). A malacological analysis. Palaeogeography, Palaeoclimatology, Palaeoecology, 59, 293-314.

ROUSSEAU D.-D., PUISSÉGUR J.-J. \& LAUTRIDOU J.-P., 1990 - Biogeography of the Pleistocene pleniglacial malacofaunas in Europe. Stratigraphic and climatic implications. Palaeogeography, Palaeoclimatology, Palaeoecology, 80, 7-23.

ROUSSEAU D.-D., 1992 - Terrestrial molluscs as indicators of global aeolian dust fluxes during glacial stages. Boreas, 21, 105-109.

ROUSSEAU D.-D., LIMONDIN N. \& PUISSÉGUR J.J. 1993 - Holocene Environmental Signals from Mollusk Assemblages in Burgundy (France). Quaternary Research, 40, 237-253.

ROUSSEAU D.-D., ZÖLLER L. \& VALET J.-P., 1998 - Late Pleistocene Climatic Variations at Achenheim, France, Based on a Magnetic Susceptibility and TL Chronology of Loess. Quaternary Research, 49, 255-263.

ROUSSEAU D.-D., ANTOINE P., HATTÉ C., LANG A., ZÖLLER L., FONTUGNE M., BEN OTHMAN D., LUCK J.M., MOINE O., LABONNE M., BENTALEB I. \& JOLLY D., 2002 - Abrupt millenial climatic changes from Nussloch (Germany) Upper Weichselian eolian records during the Last Glaciation. Quaternary Science Reviews, 21, 1577-1582.

SOMME J., LAUTRIDOU J..P., HEIM J., MAUCORPS J. PUISSEGUR J.-J., ROUSSEAU D.-D., THEVENIN A. \& VAN VLIET-LANOE B., 1986 - Le cycle climatique du Pléistocène Supérieur dans les loess d'Alsace à Achenheim. Bulletin de l'Association Française pour l'Etude du Quatemaire, 25-26, 97-104.

VAN VLIET-LANOË B., 1987 - Le rôle de la glace de ségrégation dans les formations superficielles de l'Europe de l'Ouest. Doctorat d'Etat, Univ. Paris I - Sorbonne, 854 p.

VANDENBERGHE J, HUIJZER B.S, MÜCHER H \& LAAN W., 1998 - Short climatic oscillations in a western European loess sequence (Kesselt, Belgium). Journal of Quaternary Science, 13, (5), 417-485.

VIDAL L LABEYRIE L CORTIJO E ARNOLD $M$ DUPLESSY J.-C., MICHEL E., BECQUÉ S. \& VAN WEE RING T.C.E., 1997 - Evidence for changes in the North Atlantic Deep Water linked to meltwater surges during the Heinrich events. Earth and Planetary Science Letters, 146, 13-27.

XIAO J., PORTER S.C., AN Z., KUMAI H. \& YOSHIKAWA S., 1995 - Grain size of quartz as an indicator of winter monsoon strength on the Loess Plateau of Central China during the last 130,000 yr. Quaternary Research, 43, 22-29.

ZÖLLER L., STREMME H. \& WAGNER G.A., 1988 - Thermolumineszenz-datierung an löss-paläoboden-sequenzen von nieder-, mittel- und oberrhein/Bundesrepublik Deutschland. Chemical Geology, 73, 39-62. 